\title{
Emerging role of EGFR and lung cancer treatments
}

\begin{abstract}
Tyrosine kinase inhibitors (TKIs) against targetable mutations such as epidermal growth factor receptor (EGFR) are highly efficient in treating advanced stage lung cancers. The usage of EGFR inhibitors (EGFRI) is associated with an improved response rate and improved Progression-free survival (PFS) compared to chemotherapy. Frequently expressed EGFR mutants include 19del, T790M, L858R and recently discovered secondary mutation C797S. There is also some possibility to improve outcome by focusing on better EGFRI and/or combining EGFRI with other therapies like chemotherapy, vascular endothelial growth factor (VEGF) inhibitors and immunotherapy. This review provides an overview of the current status and potential future for EGFR therapies and LUNG CANCER treatments.
\end{abstract}

Keywords: EGFR mutation, treatments, chemotherapy, lung cancer

\author{
Volume 5 Issue 5 - 2018
}

\author{
Akhilesh Rawat,' Ajay Pal Singh, ${ }^{2}$ Kumar GT,' \\ Preeti Upadhyay,' Rajeev Nema' \\ 'Molecular Diagnostics,3B BlackBio Biotech India Ltd.7-C. \\ Industrial Area, Govindpura, Bhopal, Madhya Pradesh, India \\ ${ }^{2} \mathrm{All}$ india institute medical science, Bhopal,India
}

Correspondence: Rajeev Nema, Scientist, R \& D (Molecular Diagnostics, 3B Black Bio Biotech India Ltd, 7-C, Industrial Area, Govindpura, Bhopal-462023, Madhya Pradesh, India, Tel +91 755 4077847, +91 9993425698, Fax +91 755-4282659,

Email rajeev@3bblackbio.com

Received: August II, 2018 | Published: September 17, 2018

\section{Introduction}

Cancer incidence rates are growing rapidly globally and according to the estimation from the World Health Organization (WHO), there will be 21.7 million people diagnosed with cancer. At present, cancer is the second leading cause of death around the globe. Most of the increase is expected to be concentrated in developing countries due to heavy smoking, poor diet, physical inactivity and environmental pollutions. In 2012, it was observed that the lung cancer was among the 10 most commonly founded cancers that are frequently diagnosed worldwide (Lung cancer statistics). The third-most commonly diagnosed cancer in both men and women is the lung cancer (Cancer statistics, 2017).

Since epidermal growth factor receptor discovered in 1986, the EGFR Gene has emerged as an essential factor for the development and growth of human malignancies, including lung cancer. ${ }^{1}$ The EGFR is found to be associated with the direct cell proliferation, growth, differentiation, and apoptosis. As biomarkers, EGFR is a high-ranking member as it regulates a variety of cellular processes, including cell growth, production, and progression. In fact, in multiple tumorigenic processes such as proliferation of cancer cells, angiogenesis, and metastasization, EGFR signal transduction network play a key role. Every time, EGFR aberrant activation is prognostic in NSCLC, which provided a solid foundation for the development of EGFRtargeting strategies for NSCLC. ${ }^{2}$ Prospective strategies, which inhibit the growth-promoting effect of EGFR or activate its pro-apoptotic function, are presently being explored.

\section{Lung cancer}

The main reason behind the cancer-related mortality worldwide in both men and women is the lung cancer, ${ }^{3}$ with an approximately mortality rate of 1.3 million yearly ${ }^{3}$ (National Lung Screening Trial Research, 2014). Lung cancer is divided into two parts: first, if the cancer originates in the lungs, it is termed as "primary," and if it originates elsewhere in the body, but has metastasized to the lungs, it is termed as "secondary." On the basis of appearance under a microscope, the lung cancer can be classified as follows: nonsmall cell lung cancer (NSCLC) and small cell lung cancer (SCLC). Lung cancer occurs due to oncogenes and tumor suppressor gene up regulation and down regulation. ${ }^{3,4}$ When patients with EGFR mutation nonsmall cell lung cancer (NSCLC) are treated with tyrosine kinase inhibitors (TKIs), such as gefitinib, erlotinib, and afatinib, they show improved progression-free survival (PFS) compared with standard chemotherapy. ${ }^{3,4}$ Unfortunately, patients develop resistance, including acquired EGFR mutations such as T790M and C797S. ${ }^{5}$ To combat this resistance, second- and third-generation EGFR TKIs have been developed; however, therapeutic resistance still persists. It has been observed that about NSCLC, mutations in EGFR are common. ${ }^{6}$ Frequent genetic polymorphisms and inactivation of tumor suppressor genes damage to chromosomes 3 p, 5q, 13q, and 17 p are predominantly regular in small cell lung carcinoma. ${ }^{7-10}$

\section{Lung cancer in India}

According to estimates published in The Lancet today around $5,55,000$ people died of cancer in $2010, .^{11}$ Reliable information on new cancer cases is provided by the population-based cancer registries in India and forms the basis for the national incidence estimates available in the IARC's GLOBOCAN database. In a recent study, tobaccorelated cancers represented around $42 \%$ of male and $18 \%$ of female cancer deaths. ${ }^{12}$ In India, approximately 63,000 new lung cancer cases are reported every year. ${ }^{13}$ In Bengaluru and Chennai, lung cancer is the second, and the third leading cancer site, respectively. In women, lung cancer is one of the 10 leading sites in 4 of the 6 cancer registries, i.e., in Bhopal, Chennai, Delhi, and Mumbai. ${ }^{14}$ The national cancer registry program under the Indian council of medical research, after studying the lung cancer incidence rate over 24 years (1982-2005), has found that lung cancer is the subsequent leading cause of cancer in women and has increased by a yearly percentage change of 2.7 in Bengaluru, 4.6 in Chennai, and 2 in Delhi. ${ }^{15}$

\section{EGFR mutations and lung cancer}

The molecular targeted anticancer drugs have paved a way for new treatments in the molecular pathogenesis of the lung cancer. The most established target of lung cancer EGFR. ${ }^{16}$ It is a member of the ErbB kinase family. It is structurally related receptor tyrosine kinases. ErbB proteins play a vital role in the regulation of cellular proliferation, 
and their dysregulation has been identified in a variety of cancers. ${ }^{17,18}$ For example, somatic mutations of EGFR have been reported in approximately $50 \%$ of Asian patients and $10-15 \%$ of Caucasian patients with lung adenocarcinoma, ${ }^{19,20}$ with the most common mutations in these populations being exon 19 deletions (Del19), and an L858R point mutation (L858R). The most prevalent secondary or acquired mutations in the EGFR gene are T790M and C797S, the presence of which correlates with resistance to first, second and third line of tyrosine kinase inhibitors. This Mini review describes the clinically significant EGFR gene mutations, and the efficacy of small molecule EGFR TKIs as targeted therapies for these gene mutations. Therapeutic strategies to overcome resistance, including selected emerging and novel therapies are discussed. ${ }^{21}$

\section{Conclusion}

Advanced NSCLC testing for mutations in EGFR paved the way in the treatment-decision making pathway. In Indian, NSCLC patients, EGFR TKIs either added to or given after standard chemotherapy may also improve results, but additional evidence is necessary. ${ }^{22,23}$ as a maintenance therapy after first-line, erlotinib has also shown a role in non-progressive patients alone after platinumbased chemotherapy or in combination with bevacizumab after a bevacizumab and cisplatin regimen..$^{24,25}$ In addition, wide research on individual patient's tumor biology may be necessary for appropriate targeted therapy. This individual patient approach would finally result in improved PFS and overall survival for the diverse category of lung cancer patients. EGFR-TKIs comprised a major part of the treatment strategy, especially for patients with poor PFS lung cancer in the real world. Further to this, the real challenge is therapeutic agents into the management of patients with earlier stage disease diagnosis with the hope of truly improving rates of cure for the devastating illness that is lung cancer.

\section{Acknowledgements}

The authors express gratitude Director Dhirendra Dubey 3B BlackBio Biotech India Ltd for his kind support and critical reading of the manuscript.

\section{Conflict of interest}

The author declares no conflict of interest.

\section{References}

1. Liu X, Wang P, Zhang C, et al. Epidermal growth factor receptor (EGFR): A rising star in the era of precision medicine of lung cancer. Oncotarget. 2017;8(30):50209-50220.

2. Zhong WZ, Zhou Q, Wu YL. The resistance mechanisms and treatment strategies for EGFR-mutant advanced non-small-cell lung cancer. Oncotarget. 2017;8(41):71358-71370.

3. GBD 2016 DALYs and HALE Collaborators. Global, regional, and national disability-adjusted life-years (DALYs) for 333 diseases and injuries and healthy life expectancy (HALE) for 195 countries and territories, 1990-2016: a systematic analysis for the Global Burden of Disease Study 2016. Lancet. 2017;390(10100):1260-1344.

4. Lee EY, Muller WJ. Oncogenes and tumor suppressor genes. Cold Spring Harb Perspect Biol. 2010;2(10):a003236.

5. Ko B, Paucar D, Halmos B. EGFR T790M: revealing the secrets of a gatekeeper. Lung Cancer (Auckl). 2017;8:147-159.

6. Kim JS, Cho MS, Nam JH, et al. Prognostic impact of EGFR mutation in non-small-cell lung cancer patients with family history of lung cancer. PLoS One. 2017;12(5):e0177015.
7. Chen J, Fu L, Zhang LY, et al. Tumor suppressor genes on frequently deleted chromosome $3 \mathrm{p}$ in nasopharyngeal carcinoma. Chin J Cancer. 2012;31(5):215-222.

8. Tai AL, Mak W, Ng PK, et al. High-throughput loss-of-heterozygosity study of chromosome $3 p$ in lung cancer using single-nucleotide polymorphism markers. Cancer Res. 2006;66(8):4133-4138.

9. Rom WN, Hay JG, Lee TC, et al. Molecular and genetic aspects of lung cancer. Am J Respir Crit Care Med. 2000;161(4 Pt1):1355-1367.

10. Larsen JE, Minna JD. Molecular biology of lung cancer: clinical implications. Clin Chest Med. 201;32(4):703-740.

11. Ryerson AB, Eheman CR, Altekruse SF, et al. Annual report to the nation on the status of cancer, 1975-2012, featuring the increasing incidence of liver cancer. Cancer. 2016;122(9):1312-1337.

12. Juneja A, Adhikari T, Pandey A, et al. Share of tobacco related cancers: gender and time gaps-Indian scenario. J Clin Diagn Res. 2015;9(1):LC01-LC03.

13. Noronha V, Dikshit R, Raut N, et al. Epidemiology of lung cancer in India: focus on the differences between non-smokers and smokers: a single-centre experience. Indian J Cancer. 2012;49(1):74-81.

14. Koul PA, Kaul SK, Sheikh MM, et al. Lung cancer in the Kashmir valley. Lung India. 2010;27(3):131-137.

15. Chatterjee S, Chattopadhyay A, Senapati SN, et al. Cancer registration in India - current scenario and future perspectives. Asian Pac J Cancer Prev. 2016;17(8):3687-3696.

16. Sun S, Schiller JH, Spinola M, et al. New molecularly targeted therapies for lung cancer. J Clin Invest. 2007;117(10):2740-2750.

17. Vincent MD, Kuruvilla MS, Leighl NB, et al. Biomarkers that currently affect clinical practice: EGFR, ALK, MET, KRAS. Curr Oncol. 2012;19(Suppl 1):S33-S44.

18. Wieduwilt MJ, Moasser MM. The epidermal growth factor receptor family: biology driving targeted therapeutics. Cell Mol Life Sci. 2008;65(10):1566-1584.

19. Kobayashi Y, Mitsudomi T. Not all epidermal growth factor receptor mutations in lung cancer are created equal: Perspectives for individualized treatment strategy. Cancer Sci. 2016;107(9):1179-1186.

20. Lindeman NI, Cagle PT, Beasley MB, et al. Molecular testing guideline for selection of lung cancer patients for EGFR and ALK tyrosine kinase inhibitors: guideline from the college of American pathologists, International association for the study of lung cancer, and association for molecular pathology. J Thorac Oncol. 2013;8(7):823-859.

21. Batra U, Lokeshwar N, Gupta S, et al. Role of epidermal growth factor receptor-tyrosine kinase inhibitors in the management of central nervous system metastases in epidermal growth factor receptor mutation-positive nonsmall cell lung cancer patients. Indian J Cancer. 2017;54(Suppl):S37-S44.

22. Under the aegis of Lung Cancer Consortium Asia (LCCA); Indian Cooperative Oncology Network (ICON); Indian Society of Medical \& Pediatric Oncology (ISMPO); Molecular Oncology Society (MOS) and Association of Physicians of India API). Indian consensus statement for treatment of advanced non small cell lung cancer: First line, maintenance, and second line. Indian J Cancer. 2017;54(1):89-103.

23. Bhatt AD, Pai R, Rebekah G, et al. Clinicopathologic features of nonsmall cell lung cancer in India and correlation with epidermal growth factor receptor mutational status. Indian J Cancer. 2013;50(2):94-101.

24. Gentzler RD, Patel JD. Maintenance treatment after induction therapy in non-small cell lung cancer: latest evidence and clinical implications. Ther Adv Med Oncol. 2014;6(1):4-15.

25. Mascaux C, Tomasini P, Greillier L, et al. Personalised medicine for nonsmall cell lung cancer. Eur Respir Rev. 2017;26(146). 